\title{
A resolução de problemas como prática interdisciplinar na educação: uma proposta epistemetodológica
}

\author{
The problem solving as interdisciplinary practice in education:
} an epistemetodological proposal

\section{Editores \\ Maria Inês Côrte Vitoria PUCRS, RS, Brasil Pricila Kohls dos Santos PUCRS, RS, Brasil}

\section{Equipe Editorial}

Carla Spagnolo

PUCRS, Brasil

Rosa Maria Rigo

PUCRS, Brasil

\section{ISSN 2179-8435}

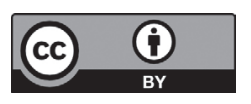

Este artigo está licenciado sob forma de uma licença Creative Commons Atribuição 4.0 Internacional, que permite uso irrestrito, distribuiçăo e reproduçấo seja corretamente citada http://creativecommons.org/licenses/by/4.0/deed.pt_BR

\author{
Altair Alberto Fávero ${ }^{a}$ \\ Carina Tonieto ${ }^{b}$ \\ Bianca Posselc
}

\section{RESUMO}

O objetivo deste texto é apresentar a resolução de problemas como uma possibilidade de prática interdisciplinar a partir de uma concepção epistemetodológica, buscando pensar alternativas metodológicas que tornem possível a experimentação da interdisciplinaridade no contexto escolar/acadêmico. A pergunta provocadora e orientadora aqui apresentada é: a concepção epistemológica-metodológica da resolução de problemas é uma alternativa promissora para a construção de práticas interdisciplinares na educação? Este estudo consiste numa pesquisa bibliográfica e exploratória. Metodologicamente, representa uma análise teórica ancorada no método analíticohermenêutico, orientada epistemologicamente por uma perspectiva racionalista, tomada em seu posicionamento crítico-analítico e enfoque da complexidade. Na primeira parte do texto, apresenta-se a concepção epistemológica de Popper (2013; 2008; 2004; 1975), ancorada na proposta do método de conjecturas e refutações que se baseia na criação e resolução de problemas, a partir do qual é possível a construção e reconstrução do conhecimento; e, na segunda parte, apresenta-se a proposta epistemetodológica da colocação e resolução de problemas como possibilidade de prática interdisciplinar nos processos de ensino e aprendizagem, amparados em Pozo (2008)

\footnotetext{
a Pós-Doutorado na Universidad Autónoma del Estado de México (UAEMéx), Doutor em Educação (UFRGS). Atua como professor e pesquisador no Curso de Filosofia, no Mestrado e Doutorado em Educação da Universidade de PassoFundo, RS, Brasil (UPF).

b Doutoranda em Educação no Programa de Pós-Graduação em Educação da Universidade de Passo Fundo, RS, Brasil (UPF). Atua como professora no curso de Filosofia e na área de Ética e Conhecimento na UPF e no Instituto Federal de Educação, Ciência e Tecnologia do Rio Grande do Sul (IFRS, Campus Ibirubá). Coordenadora do Curso de Filosofia na UPF. E-mail: <tonieto.carina@ gmail.com>.

c Graduanda do curso de Filosofia da Universidade de Passo Fundo e bolsista CNPq do Projeto de Pesquisa Interdisciplinaridade, Docência Universitária e Políticas Educacionais, coordenado pelo professor doutor Altair Alberto Fávero. E-mail: <bianca.possel@ gmail.com>
} 
e Sommermman (2015). A conclusão é que a proposta epistemetodológica da resolução de problemas é uma alternativa promissora para a construção de práticas interdisciplinares na educação, devido ao potencial criativo. No entanto, não representa apenas a aquisição de ferramentas e procedimentos capazes de gerar soluções quando aplicados em si mesmos e por si mesmos, já que enquanto método precisa estar associado aos conteúdos de aprendizagem.

Palavras-chave: Interdisciplinaridade; Educação; Método; Epistemologia

\section{ABSTRACT}

The purpose of our text is to present problem solving as a possibility of interdisciplinary practice from an epistemetodological conception, seeking think methodological alternatives that make possible the interdisciplinary experimentation in the school / academic context. The provocative and guiding question of our study was: the epistemological-methodological conception of problem solving is a promising alternative for the construction of interdisciplinary practices in education? Our study consisted of a bibliographical and exploratory research. Methodologically it consists of a theoretical analysis anchored in the analytical-hermeneutical method, oriented epistemologically by a rationalist perspective, taken in its critical-analytical positioning and complexity focus. In the first part of the text we present the epistemological conception of Popper $(2013 ; 2008 ; 2004 ; 1975)$ anchored in the proposed method of conjectures and refutations consisting in the creation and resolution of problems, from which it is possible the construction and reconstruction of knowledge; and in the second part we present the epistemetodological proposal of the placement and resolution of problems as a possibility of interdisciplinary practice in the teaching processes and learning, supported by Pozo (2008) and Sommermman (2015). Our conclusion is that the epistemetodological proposal of problem solving is a promising alternative for the construction of interdisciplinary practices in education, due to the creative potential, however, does not consist in the acquisition of tools and procedures capable of generating solutions when applied in themselves and by themselves, since as a method it must be associated to the learning contents.

Keywords: Interdisciplinarity; Education; Method; Epistemology

[...] as teorias são redes lançadas para capturar aquilo que denominamos 'o mundo': para racionalizá-lo, explicá-lo, dominá-lo.

Nossos esforços são no sentido de tornar as malhas da rede cada vez mais estreitas.

(POPPER, 2013, p. 53) 


\section{Considerações iniciais}

$\mathrm{P}$

ropõe-se no presente texto apresentar a resolução de problemas como uma possibilidade de prática interdisciplinar a partir de uma concepção epistemetodológica, buscando pensar alternativas metodológicas que tornem possível a experimentação da interdisciplinaridade no contexto escolar/acadêmico. Parte-se da concepção defendida por Bachelard (1977) e Popper (1975) de que a experiência é sempre informada pela teoria, ou seja, é esta que orienta o olhar do investigador/professor na colocação e resolução de problemas. Para o primeiro, toda produção do conhecimento é informada teoricamente e nunca neutra, o que implica reconhecer que o pesquisador/professor não encontra problemas, mas os constrói a partir de determinados referenciais teóricos; o segundo afirma que a ciência trabalha com problemas que são teóricos e, por consequência, a produção do conhecimento é resultado de uma tentativa teórica de resolver problemas que o próprio pesquisador/professor coloca, como sujeito epistemológico, e precisa apontar soluções, as quais são sempre provisórias. Nesse sentido, para Popper (1975), também, a colocação de problemas de pesquisa é sempre informada teoricamente, a partir do 'horizonte de expectativas' pelo qual o sujeito atribui significado às suas experiências, ações e observações. Assim, é a teoria que permite colocar problemas, aponta para onde ter um interesse, o que e como observar e dá parâmetros para análise dos resultados.

Porém, tanto Bachelard (1977) quanto Popper (1975) reconhecem que as questões pessoais/subjetivas ou psicológicas do investigador/professor estão presentes no processo de produção do conhecimento, ou seja, todo sujeito conhecedor trabalha a partir de seus referenciais pessoais e ideológicos, uma vez que é um ser social. No entanto, quando estas não são devidamente consideradas no processo de produção do conhecimento, podem conduzir a dois equívocos: ou tentar justificar o conhecimento a partir de suas crenças pessoais e considerar conhecimento somente aquilo que está de acordo com uma determinada ideologia; ou tentar eliminar todo e qualquer vestígio de subjetividade do processo de conhecer, como intentava o positivismo. Ambas as posições correm o risco de cair no totalitarismo das ideias e na instrumentalização formalista. Para Bachelard (1977), o exercício da vigilância epistemológica é central para evitar tais equívocos, por permite ao pesquisador/professor a "vigilância de si", ou seja, a capacidade de analisar criticamente a si mesmo e seus processos de tomada de decisão em relação a seu objeto de estudo; a "vigilância ao quadrado", que consiste na vigília na aplicação do método; e a "vigilância ao cubo", que é o momento de colocar à prova o próprio método. Tais atitudes vigilantes são constituintes dos processos em comunidades, por isso, inerentes ao processo de discussão, ação e avaliação crítica entre pares. Segundo Popper (1975), o exercício da crítica

\footnotetext{
1 Consiste na vigilância da vigilância, representada por Bachelard (1977, p. 92) pela notação exponencial (vigilância) ${ }^{2}$.

2 Consiste na vigilância da vigilância da vigilância, representada por Bachelard (1977, p. 92) pela notação exponencial (vigilância) ${ }^{3}$.
} 
consiste em submeter as teorias a testes intersubjetivos, já que nesse processo as teorias precisam ser justificadas pela sua capacidade de resolução dos problemas por meio de argumentos lógicos e não mediante argumentos de convencimento de cunho pessoal/subjetivo.

Levando em consideração tais concepções, é que se recorre ao uso da categoria epistemetodológica, utilizada por Tello (2012) para mostrar que as escolhas metodológicas do pesquisador/professor estão ligadas às concepções epistemológicas, isto é, a opção por essa metodologia é decorrente de uma concepção a respeito do conhecimento e seus processos de construção. Por isso, para justificar a resolução de problemas como uma prática interdisciplinar, fazse uso, primeiramente, das considerações epistemológicas de Popper, para posteriormente apontá-la como alternativa metodológica para a educação, amparada em Pozo (1998).

Este estudo busca construir uma resposta satisfatória para a pergunta: a concepção epistemológica-metodológica da resolução de problemas é uma alternativa promissora para a construção de práticas interdisciplinares na educação? Para tal, realizou-se uma pesquisa bibliográfica quanto aos seus procedimentos, e exploratória quanto aos seus objetivos. Metodologicamente, consiste numa análise teórica ancorada no método analítico-hermenêutico, orientada epistemologicamente por uma perspectiva racionalista, tomada em seu posicionamento crítico-analítico e enfoque da complexidade.

Na primeira parte do texto, apresenta-se a concepção epistemológica de Popper $(2013 ; 2008 ; 2004 ; 1975)$, ancorada na proposta do método de conjecturas e refutações que consiste na criação e resolução de problemas, a partir do qual é possível a construção e reconstrução do conhecimento; e, na segunda parte, apresenta-se a proposta epistemetodológica da colocação e resolução de problemas como possibilidade de prática interdisciplinar nos processos de ensino e aprendizagem, amparados em Pozo (2008) e Sommermman (2015).

A construção do problema, assim como as alternativas criadas para o seu enfrentamento, aconteceram no grupo de pesquisa Interdisciplinaridade, Docência Universitária e Políticas Educacionais e na disciplina Leituras Dirigidas, realizada no primeiro semestre de 2016, ambos vinculados ao Programa de Pós-Graduação em Educação da Universidade de Passo Fundo/RS/Brasil.

\section{O método das conjecturas e refutação de Karl Popper e a resolução de problemas}

Para Popper (2008, p. 80), a ciência tem sua origem nos mitos e na crítica destes, pois a sua gênese não está na coleção de observações e galeria de experimentos, mas, pelo contrário, na atitude crítica diante de técnicas e práticas ilusionistas. A tradição científica e a pré-científica possuem um elo, ou seja, ambas deixam como herança 
suas teorias, porém somente a científica deixa, também em seu legado, a atitude crítica em relação a estas. Desse modo, "as teorias são transferidas não como dogmas, mas acompanhadas por um desafio para que sejam discutidas e se possível aperfeiçoadas". Assim, é possível se evitar a instituição dos dogmas da ciência por meio da atitude crítica (razoável e racional), que consiste na "tradição de livre debate sobre as teorias para identificar seus pontos fracos e aperfeiçoá-los" (POPPER, 2008, p. 80). As teorias, desse modo, nada mais são que tentativas de explicação e resolução de determinados problemas, as quais devem ser permanentemente submetidas à crítica a fim de avaliar seu potencial explicativo.

Considerando que, como ser humano, carrega-se a possibilidade de conhecer e explicar determinados problemas que se enfrentam e que, para isso, recorre-se a leis e teorias; e que se, enquanto pesquisadores/professores, essa é a tarefa, o procedimento racional é o mais adequado a ser adotado. Na visão de Popper $(2008$, p. 81), este consiste no "método das tentativas - da conjectura e da refutação" (grifos do autor), o que implica propor "teorias ousadamente; tentar refutá-las; aceitá-las tentativamente, se fracassarmos". Assim, é por meio de tentativas provisórias (hipóteses, pressuposições e teses) de resolução de problemas que se busca construir explicações razoáveis e racionais, as quais são constantemente submetidas à crítica de modo a tentar refutá-las, e não, simplesmente, afirmá-las como verdades absolutas e perenes. Sem esse processo de crítica ou tentativa de refutação, não é possível saber em quais aspectos uma teoria e seu potencial explicativo têm de ser melhorados ou modificados.

Como se trabalha com tentativas de explicação (conjecturas e refutações), estas podem conter erros, apesar de o pesquisador/professor trabalhar arduamente (conscientemente e cuidadosamente) na tentativa de refutar sua teoria, recorrendo aos melhores argumentos e aos testes experimentais mais severos que sua teoria e astúcia lhe permitam preparar $^{3}$. A dimensão positiva do erro, ressaltada pelo método de tentativas, consiste na possibilidade de este ser apontado por meio da crítica. O que permite que seja revisto, e com isso que as teorias se modifiquem, reconstruindose em novas bases. No entanto, é preciso reconhecer que: (i) a solução de um problema é sempre provisória, uma vez que "as melhores soluções - aquelas capazes de resistir à crítica mais severa das mentes mais brilhantes e engenhosas - logo darão origem a novas dificuldades, a novos problemas" (POPPER, 1975, p. 236), porém é tal processo que permite a retificação dos problemas e a reconstrução do conhecimento em novas bases; (ii) apesar de a construção do conhecimento ser um processo de crescimento contínuo e sofrer com algumas paradas, este progride por trabalhar

\footnotetext{
3 Popper (1975, p. 34-35), para ilustrar melhor seu argumento a respeito do erro, faz uma comparação entre Einstein e uma ameba: "Einstein busca conscientemente a eliminação do erro. Ele procura matar suas teorias; é conscientemente crítico de suas teorias, as quais, por isso, procura formular nitidamente e não vagamente. Mas a ameba não pode ser crítica vis-à-vis porque não pode enfrentar suas hipóteses; estas fazem parte dela" (grifos do autor).
}

Educação Por Escrito, Porto Alegre, v. 9, n. 1, p. 41-53, jan.-jun. 2018 
com tentativas de resolução de problemas: "O crescimento do conhecimento marcha de velhos problemas para novos problemas, por meio de conjecturas e refutações" (POPPER, 1975, p. 236).

No entanto, é reconhecido o fato de que a solução dos problemas se depara com um grande número de teorias, que podem ser boas ou más, apesar de o interesse ser sempre elaborar uma boa teoria. Essa elaboração inicia-se com uma primeira tentativa, entretanto a sua qualificação é possível por meio de testes, que consistem na aplicação reiterada do "método crítico, de modo a eliminar muitas teorias inadequadas e inventando muitas teorias novas" (POPPER, 2008, p. 85). O método crítico baseia-se em conjecturar e refutar. Isso posto, significa que:

(i) Inicialmente, trabalha-se com um problema ou dificuldade a respeito do qual não se sabe muito e para o qual ainda não é possível produzir uma solução adequada.

(ii) Reconhecida a necessidade de conhecer mais a respeito do problema, aparece a dúvida: como?

(iii) Produzindo uma solução inicial e a criticando, podem-se compreender as dificuldades e o que e por que funciona ou não.

(iv) Assim, é possível passar para soluções melhores, desde que se tenha capacidade crítica para produzir sempre novas suposições (POPPER, 1975, p. 237). A capacidade crítica, no entanto, pressupõe abertura e disposição para a mudança.

A atividade de compreensão opera, desse modo, por meio de conjecturas e refutações e pode ser representada por um "esquema geral de solução de problemas pelo método de conjecturas imaginativas e de crítica" (POPPER, 1975, p. 159), o qual na sua forma mais simples é:

$$
\mathrm{P}_{1} \rightarrow \mathrm{TT} \rightarrow \mathrm{EE} \rightarrow \mathrm{P}_{2}
$$

Onde: $\mathrm{P}_{1}$ é o problema de partida; TT é a teoria experimental ou a primeira solução conjectural imaginativa alcançada; EE é a eliminação do erro, que consiste em um rigoroso exame crítico da conjectura proposta ou de testes experimentais, se houver mais de uma conjectura disponível, e representa também uma discussão crítica e avaliação comparativa das teorias concorrentes; $\mathrm{P}_{2}$ é a situação-problema que emerge da primeira tentativa crítica de solucionar o problema inicial, que leva a uma segunda tentativa e assim sucessivamente. A compreensão satisfatória é alcançada quando "a interpretação, a teoria conjectural, encontra apoio no fato de explicar muitos subproblemas, alguns dos quais não foram vistos no começo", podendo avaliar seu progresso "comparando $\mathrm{P}_{1}$ com alguns de nossos problemas posteriores $\left(\mathrm{P}_{11}\right.$ digamos)" (POPPER, 1975, p. 160). Assim, "podemos aprender com os erros que cometemos" (POPPER, 2008, p. 17). 
Nesse processo, assumem um papel modesto, tanto a razão quanto a observação. A razão, que por meio de argumentos racionais, tem a função de criticar as tentativas por vezes equivocadas de resolução de problemas; e a experiência, que por meio da observação tem a função de experimento que pode ajudar na identificação dos erros. $O$ conhecimento e em decorrência o conhecimento científico progridem por meio de tentativas de solução de problemas ou conjecturas. Estas não são a expressão do subjetivismo dos sujeitos, mas controladas pelo espírito crítico e consistem nas tentativas de refutação, compostas de testes severamente críticos.

As conjecturas podem vencer os testes ou não. Quando vencem, não significa que encontraram a verdade definitiva e por isso são justificadas no sentido positivo, nem que são prováveis no sentido do cálculo probabilístico, mas que no momento são as que melhor se aproximam de uma solução adequada para o problema proposto, levando em consideração as teorias concorrentes. Ou seja, são verossímeis, porém continuam abertas para novas revisões ou colocação de novos problemas; quando refutadas, significa que não produziram uma resposta satisfatória ao problema proposto, o que não implica que devam ser eliminadas, mas revistas.

O caráter de abertura para novas revisões é uma característica intrínseca ao processo de resolução de problemas por meio de conjecturas e refutações, uma vez que trabalha com tentativas examinadas criticamente que evidenciam infinitamente os erros e, por consequência, apontam as dificuldades inerentes ao processo de colocação e resolução de problemas. Entretanto, é por meio desse processo que acontece a familiarização com os problemas e são possíveis propostas de solução cada vez melhores, evidenciando o aspecto positivo do erro. Este possibilita o aprendizado e a expansão do conhecimento, porém está sempre aberto a revisões, o que implica, partindo dessa concepção, uma atitude de aceitação da incerteza e da negação de uma atitude autoritária e pretensiosa a respeito do que se conhece. É nesse espaço de colocação de problemas, tentativa de solução e avaliação crítica que se identifica o potencial criativo e crítico da interdisciplinaridade, pois esta estaria presente em todas as fases do processo, promovendo a interação contínua e coordenada das diversas áreas do conhecimento, já que compreendida como uma atitude epistemetodológica, a qual é apresentada a seguir.

\section{A prática interdisciplinar como colocação e resolução de problemas na educação}

Como lembra Becker (1993), as ações ligadas ao ensino e à aprendizagem são amparadas e justificadas por concepções epistemológicas, isto é, concepções de conhecimento e de como se dá o seu processo de construção. É nessa linha que se vincula a concepção de Popper a respeito da produção do conhecimento, ou seja, do método de 
resolução de problemas por meio de conjecturas e refutações, como um aliado na busca por alternativas metodológicas para a prática interdisciplinar na educação.

Considerando-se a definição de interdisciplinaridade dada por Sommermann (2015, p. 208), na qual a "interação prolongada e coordenada" entre as disciplinas tem como objetivo a "resolução de determinado problema complexo" que não poderia ser resolvido pela via monodisciplinar, então pode-se afirmar que o método da resolução de problemas de modo crítico, apontado por Popper, é uma possibilidade viável aliada ao exercício da criatividade.

Quando se percebe que as práticas disciplinares, seja no ensino, na pesquisa ou na extensão, não conseguem mais dar conta de produzir determinadas respostas para os problemas a serem investigados, então se está reconhecendo a necessidade de novos arranjos entre as disciplinas. Uma das possibilidades é construir problemas por meio de conjecturas estáveis ${ }^{4}$, trabalhando de modo cooperativo e colaborativo, a fim de propor novos olhares para os problemas, assim como novas soluções e possibilidades de aplicação. Cabe lembrar que Popper (2008, p. 144) já chamava atenção para o fato de que "as teorias são nossas invenções, nossas ideias - não se impõem a nós, são instrumentos de pensamento que fabricamos", e essa fabricação pode ser resultado de uma interação interdisciplinar. Entretanto, todo esse processo é possível a partir da colocação de um problema que fomente a possibilidade de resolução interdisciplinar. Nesse sentido, Somermann $(2015$, p. 209) afirma que o primeiro passo para a efetivação da interação entre as disciplinas é a definição do problema 5 .

Para Pozo (1998), um dos maiores desafios dos processos pedagógicos é fazer com que os estudantes aprendam a aprender, uma vez que é por meio desse processo que conseguirão resolver os mais variados problemas que surgem numa sociedade que se modifica constantemente e que, automaticamente, exige tanto esforço de resolver quanto de identificar problemas. Problemas estes que, por vezes, são diferentes dos apresentados na escola, e, por isso, os estudantes precisam construir novos conhecimentos e estratégias a fim de serem "pessoas capazes de enfrentar situações e contextos variáveis, que exijam deles a aprendizagem de novos conhecimentos e habilidades" (POZO, 1998, p. 9). Esse processo de aprender a aprender é possível por meio da apresentação de situações problemáticas aos estudantes, para as quais precisam propor soluções criativas. Assim, o desafio é encontrar "por si mesmos respostas às perguntas que os inquietam ou precisam responder, ao invés de esperar uma resposta já elaborada por outros e transmitida pelo livro-texto ou pelo professor" (POZO, 1998, p. 9).

4 Conjecturas estáveis para Popper (2008, p. 144) são aquelas que se referem à realidade ou afirmam algo a respeito da realidade e que, enquanto teorias testáveis, implicam a previsão de ocorrência de determinados eventos.

5 Somermann faz essa afirmação amparado em Klein e Repko. 
A criação e a solução de problemas é uma das ferramentas que pode ser utilizada para instigar a aprendizagem autônoma dos estudantes. No entanto, o que é considerado um problema para o professor pode não fazer sentido para os estudantes, por isso não basta saber resolver problemas, é preciso aprender a formulá-los também. Para ilustrar essa situação, Echeverría e Pozo (1998, p. 13) contam a história de um professor que ao perguntar para um de seus estudantes negros quantas pernas têm um gafanhoto, recebeu a resposta de que quem dera ele ter os mesmos problemas que seu professor. Enquanto o professor tinha um problema de cunho intelectual que julgava ser importante, a resposta do estudante revela que o problema proposto não era de tamanha importância para si, uma vez que enfrentava problemas de outra natureza. Nesse sentido, concorda-se com Echeverría e Pozo (1998, p. 15): não se trata apenas de ensinar os estudantes a resolver os problemas propostos pelo professor, mas de prepará-los para que formulem problemas a partir de suas experiências de vida para os quais buscarão soluções. Nas palavras dos autores: "Não é uma questão de somente ensinar a resolver problemas, mas também de ensinar a propor problemas para si mesmo, a transformar a realidade em um problema que mereça ser questionado e estudado".

Uma situação pode ser caracterizada como problemática quando quem se depara com ela a reconheça como tal e não consiga resolvê-la automaticamente. Para serem resolvidos, os problemas precisam ser refletidos e, também, é necessário que se façam projeções de possíveis soluções ou erros que podem ocorrer no processo de resolução. Por isso, um exercício se difere de um problema. O exercício, para ser resolvido, necessita da aplicação ou replicação de uma regra, que após entendida passa a ser usada quase que automaticamente. Consertar um computador, um chuveiro ou um carro, pode ser um exercício comum no dia a dia de pessoas que já têm o conhecimento para fazê-lo e o fazem sempre (exercício), mas, para outras que não têm conhecimento prévio e nunca consertaram algo do tipo, essa situação se torna um problema complicado. Qual é, então, a relação entre um exercício e um problema?

O exercício exige a aplicação da regra que foi aprendida anteriormente e segue um padrão de resolução; o problema exige a mobilização e a combinação de um conjunto de conhecimentos e suas regras de aplicação para que se possa propor uma solução satisfatória. Quando um problema novo é proposto para os estudantes, é comum que recorram a conhecimentos anteriormente exercitados para tentar solucionar, porém é a impossibilidade de resolvê-lo de maneira padronizada que coloca desafios e exige a mobilização de outros conhecimentos e técnicas. No entanto, se o mesmo problema for proposto e resolvido diversas vezes, acaba se tornando um exercício. Sendo assim, um problema pode vir a ser um exercício na medida em que este é exercitado repetidamente; já um exercício não pode vir a ser um problema, mas pode ajudar a solucionar novos problemas.

Geralmente disciplinar ou multidisciplinar, o exercício está centrado na capacidade de aplicação de um determinado conhecimento para encontrar uma solução correta que já está dada. O problema, por sua vez, exige a combinação de vários conhecimentos para a proposição de uma solução, o que caracteriza o seu potencial 
interdisciplinar. Nessa direção, concorda-se com Echeverría e Pozo (1998) no sentido de que a prática de criar e solucionar problemas deve ser incentivada em todas as áreas do conhecimento, pois, toda vez que o estudante se depara com um problema novo, as possibilidades de resolução são muito maiores e mais criativas. Isso porque podem fazer novas ligações entre os problemas e, também, novas combinações ou arranjos entre os conhecimentos das diferentes disciplinas.

Segundo Echeverría e Pozo (1998), os problemas podem ser classificados de múltiplas formas. Uma destas pode ser feita levando em consideração o método utilizado para a resolução, no qual se tem problemas dedutivos e problemas indutivos. Os problemas dedutivos geralmente são os que se referem às ciências exatas e da natureza (como matemática, física, química, etc.). São os problemas para os quais se pode ter certeza quanto à correção da resposta e ao porquê, pois se trata da aplicação de regras gerais exatas em uma situação mais específica. Por isso, podem facilmente ser identificados como exercícios. Já os problemas indutivos são característicos das ciências humanas (filosofia, sociologia, psicologia, etc.), pois se trata do processo de extrair novas conclusões a partir das informações conhecidas e disponíveis. Porém nem sempre é possível encontrar a resposta correta, assim como se admitem várias respostas ou soluções. Trata-se de buscar soluções mais ou menos adequadas, dependendo do contexto, das condições estabelecidas pelos problemas e dos conceitos utilizados como referência. Nessa classificação, identificou-se um potencial interdisciplinar em ambos os tipos de problemas, pois podem demandar a elaboração de arranjos entre os conhecimentos de diversas disciplinas, seja para encontrar a resposta certa, seja para propor uma ou mais soluções satisfatórias. No entanto, no segundo tipo de problema é que se identificou maior potencial para a criação de soluções mais críticas e criativas, pela necessidade de arranjos entre os conhecimentos disciplinares.

Outra classificação, apresentada pelos autores, utiliza como método a forma de elaboração do problema. Segundo esse critério, os problemas podem ser bem definidos ou mal definidos. São bem definidos quando se consegue entender exatamente o que precisa ser feito até chegar à sua resolução. Os exercícios sempre serão bem definidos, pois se sabe de onde se está partindo, o que é preciso fazer e aonde se deve chegar. Os problemas mal definidos, por sua vez, não são ruins ou não merecem atenção, bem pelo contrário, são problemas que exigem muito cuidado no processo de resolução, já que podem conter mais de uma solução. E talvez não seja possível encontrar uma solução plenamente satisfatória, visto que pode envolver conceitos e conhecimentos que estão em processo constante de reelaboração. Nesse modo de diferenciação dos problemas, percebe-se o potencial interdisciplinar, porém nos segundos parece residir a maior complexidade e necessidade de reflexão crítica.

Durante o processo da resolução de problemas, a clareza do método a ser utilizado para chegar à solução é fundamental, pois ajuda a esclarecer o próprio problema e, por consequência, torna mais fácil sua resolução. Porém, nem sempre se consegue descrever de modo claro aquilo que se faz. Essa dificuldade em explicar os procedimentos 
para a solução do problema pode residir também no professor, porém é ele quem deve auxiliar e orientar os estudantes para que consigam aprender a aprender. Da mesma forma que não se ensina uma pessoa a andar de bicicleta apenas descrevendo quais são os movimentos adequados, também não se ensina a criar e resolver problemas somente descrevendo os procedimentos, pois a tentativa é uma dimensão importante. Aprende-se a andar de bicicleta, andando de bicicleta; aprende-se a resolver problemas, resolvendo problemas; entretanto, em ambas as aprendizagens, é muito importante o auxílio recebido durante o processo. Desse modo, o professor não ensina a criar/elaborar e resolver problemas de modo interdisciplinar, se não souber fazê-los.

O método utilizado para resolver ambos os tipos de problemas é muito semelhante ao empregado pelos cientistas, que, independentemente do conteúdo ou do nível de dificuldade que se apresenta, possui uma estrutura. Pozo e Crespo (1998, p. 72) descrevem as fases do método científico como observação e proposição de um problema, formulação de hipóteses, planejamento e execução de experiências e confronto de hipóteses; e as fases de resolução de um problema como compreensão do problema, concepção de um plano, execução do plano e análise da solução obtida. Ambas as descrições estão alinhadas ao método de conjecturas e refutações proposto por Popper, isto é, diante de um problema se projetam soluções que não são automáticas, mas construções informadas teoricamente (construídas a partir dos conhecimento disponíveis); posteriormente, elabora-se um plano rigoroso para o exame crítico da conjectura proposta, que são os testes; a melhor solução será a que resistiu aos testes, ou tentativas de refutação, sendo aceita como válida até que surjam soluções melhores. Tal método ${ }^{6}$ pode ser aprendido e aperfeiçoado.

A diferença na resolução de problemas entre os especialistas e iniciantes, para Echeverría e Pozo (1998), é que os primeiros partem para a solução especializada por possuírem mais eficiência na aplicação de procedimentos técnicos, porém tendem a propor soluções menos criativas. Os principiantes e pessoas com menos experiência tendem a trabalhar a partir da redução da diferença entre o estado inicial do problema e a solução, ou seja, reconhecido o problema, identificam uma solução e buscam os meios necessários para chegar a esta, no entanto, são mais criativos. Por isso, a aprendizagem do método ajuda os principiantes a aprimorarem suas estratégias de reconhecimento e projeção de soluções para os problemas. No entanto, a aprendizagem do método pode se converter num mero procedimento a ser repetido e, assim, transformado num exercício; ou pode ser potencializada por meio da elaboração e resolução de problemas que instiguem a construção de novos arranjos entre os conhecimentos ou disciplinas.

O grande desafio de ensinar o método da resolução de problemas é que este não deve ser visto como uma fórmula exata que deve ser seguida sem objeções, mas como uma orientação. Sua importância deve ser entendida não só para

\footnotetext{
6 O potencial interdisciplinar não está na simples aplicação do método, mas nos arranjos necessários entre os conhecimentos para a proposição de uma resolução satisfatória para o problema.
} 
a resolução de problemas matemáticos, filosóficos, químicos, biológicos, mas, também, para a vida cotidiana. Nessa direção, concorda-se com Castillo (1998, p. 107): “A presença da solução de problemas no ensino costuma tomar duas formas essenciais: como um fim em si mesma, ou seja, como o próprio objeto da aprendizagem, ou como meio para a aquisição de outros conhecimentos". Isto é, o método é também objeto de aprendizagem e pode se tornar um meio para a construção de novos conhecimentos. Como se trata de uma aprendizagem, que não tem um fim em si mesma, o método de resolução de problemas interdisciplinares pode se tornar uma alternativa viável para a realização de experiências escolares interdisciplinares, pois está aberto e comporta as mais diversas propostas de interação e integração entre as disciplinas.

\section{Considerações finais}

O método da resolução de problemas não consiste na aquisição de ferramentas e procedimentos capazes de gerar soluções quando aplicados em si mesmos e por si mesmos, assim como não garantem a efetividade dos processos de produção do conhecimento e de ensino e aprendizagem. Precisa estar associado às ferramentas conceituais, ou seja, aos conteúdos de aprendizagem, pois não tem um fim em si mesmo. Nesse sentido,conclui-se que, compreendendo o processo de construção do conhecimento como uma tentativa de resolução de problemas, que afetam as pessoas e as suas formas de vida, não se aprende apenas a resolver problemas escolares, mas também problemas cotidianos. No entanto, os problemas cotidianos ou da vida não são organizados em disciplinas como os escolares. A vida é interdisciplinar, e o desafio é se aproximar dela.

Nessa direção, pontua-se o potencial criativo da resolução de problemas como uma proposta epistemetodológica que suporta novas formas de pensar, projetar e agir no cotidiano das instituições de ensino. No entanto, aos responsáveis pela condução e orientação de tal processo, o desafio é se tornarem sujeitos interdisciplinares, já que não se ensina aquilo que não se sabe. A velha desculpa de que o papel das instituições do ensino é apontar as diversas faces e facetas do conhecimento e que cabe aos estudantes fazer a síntese e a integração entre eles, aplicando-os na resolução de problemas, não se sustenta. Isso porque, tanto o método quanto o conteúdo são objetos de aprendizagem que, pelo menos inicialmente, precisam de uma condução ou orientação. Desse modo, para que tal proposta epistemetodológica seja viável e factível, o pesquisador/professor precisa ser um sujeito criador e solucionador de problemas interdisciplinares, o que implica, por sua vez, formar-se e reformar-se. Pois, como lembra Bachelard (1996), de nada adiante uma cabeça cheia, porém fechada; e, como ressalta Popper (2008), não se trata de encontrar verdade última definitiva, mas de apresentar possibilidades de compreensão que possam ser constantemente melhoradas. 


\section{Referências}

ABBagnano, N. Dicionário de filosofia. 5. ed. São Paulo: Martins Fontes, 2007.

BACHELARD, G. O racionalismo aplicado. Rio de Janeiro: Zahar Editores, 1977.

BACHELARD, G. A formação do espírito científico: contribuições para uma psicanálise do conhecimento. Rio de Janeiro: Contraponto, 1996.

BECKER, F. A epistemologia do professor: o cotidiano da escola. Petrópolis: Vozes, 1993.

CASTILLO, J. D. A solução de problemas nos estudos sociais. In: POZO, J. I.; (Org.). A solução de problemas: aprender a resolver, resolver para aprender. Porto Alegre: Artmed, 1998, p. 103-137.

ECHEVERRÍA, M. D. P. P.; POZO, J. I. Aprender a resolver problemas e resolver problemas para aprender. In: POZO, J. I.; (Org.). A solução de problemas: aprender a resolver, resolver para aprender. Porto Alegre: Artmed, 1998. p. 13-41.

POPPER, K. R. Conhecimento objetivo: uma abordagem evolucionária. Belo Horizonte: Itatiaia: São Paulo Editora da Universidade de São Paulo, 1975.

POPPER, K. R. A lógica das ciências sociais. 3. ed. Rio de Janeiro: Tempo Brasileiro, 2004.

POPPER, K. R. Conjecturas e refutações: o progresso do conhecimento científico. 5. ed. Brasília: Editora da Universidade de Brasília, 2008.

POPPER, K. R. A lógica da pesquisa científica. 2. ed. São Paulo: Cultrix, 2013.

POZO, J. I. A solução de problemas: aprender a resolver, resolver para aprender. Porto Alegre: Artmed, 1998.

POZO, J. I.; GOMÉZ CRESPO, M. A. A solução de problemas nas ciências da natureza. In: POZO, J. I.; (Org.). A solução de problemas: aprender a resolver, resolver para aprender. Porto Alegre: Artmed, 1998. p. 67-98.

SOMMERMAN, A. Objeto, método e finalidade da interdisciplinaridade. In: PHILIPPI JR., A.; FERNANDES, V.; (Org.). Práticas da interdisciplinaridade no ensino e na pesquisa. Barueri/SP: Manole, 2015. p. 165-212.

TELLO, C. Las epistemologias de la política educativa: vigilancia y posicionamento epistemológico del investigador en política educativa. Práxis Educativa, Ponta Grossa, v. 7, n. 1, p. 53-66, jan.-jun. 2012.

Recebido em: setembro/2017

Aceito em: abril/2018

Endereço para correspondência:

Altair Alberto Fávero <favero@upf.br>

Av. Brasil Leste, 285 - São José

99052-900, Passo Fundo, RS, Brasil 